SECTION 4. Computer science, computer engineering and automation.

Marler Maukenovich Ilipov doctoral student in the specialty "6D070400-Computing Equipment and Software" of L.N. Gumilyov Eurasian National University, Kazakhstan ilipov@gmail.kz

Ayman Serikovna Iskakova

Candidate of Physical and Mathematical Sciences, Associate Professor of Department of Fundamental Mathematics of L.N. Gumilyov Eurasian National University,

Kazakhstan ayman.astana@gmail.com

\title{
MECHANISM OF OPTIMAL CHOICE OF CASE - BASED REASONING THE SITUATIONAL VECTOR WITH QUANTITATIVE COORDINATES
}

Abstract: This paper describes algorithms for computing the membership function of case - based reasoning and the choice of case-based reasoning the situational vector with quantitative coordinates with an example in the system of RFID is submitted.

Key words: case-based reasoning, the matrix of knowledge, minimax method.

\section{УДК 517.11}

\section{МЕХАНИЗМ ОПТИМАЛЬНОГО ВЫБОРА ПРЕЦЕДЕНТА ПРИ НАБЛЮДЕНИИ СИТУАЦИОННОГО ВЕКТОРА С КОЛИЧЕСТВЕННЫМИ КООРДИНАТАМИ}

Аннотация: В работе описань алгоритмы вычисления функции принадлежности прецедента и выбора прецедента при наблюдении ситуащионного вектора с количественными координатами с примером применения в системе работы RFID.

Ключевые слова: прецедент, матрица знаний, метод минимакса.

Процесс оперативного вывода по прецеденту является достаточно сложным и неоднозначным. Сложность оперативного вывода по прецеденту и прогнозирования его последствий усугубляется тем, что этот процесс практически всегда осуществляется в условиях действия факторов неопределенности и риска. Основной задачей управления интегральными схемами, например, чипами или микрочипами, является обеспечение помехоустойчивости, решение которой в условиях перекрываемости сигналов весьма затруднено [1, с.35].

Как известно (см., например, [2, с. 172]), для интеллектуальной поддержки принятия решений на основе прецедентов проблемных ситуаций используется один из следующих типов правил: правила распознавания класса прецедентов, к которому следует отнести текущую проблемную ситуацию.

В данной статье представляется подход к организации оперативного вывода по прецеденту на основе формализации матрицы знаний.

Ранее в работе Прохорова М. Д. и Федунова Б.Е. [3, с. 62] был введен алгоритм выбора прецедента при наблюдении ситуационного вектора с количественными координатами. Основным содержанием от результатов работы [3, с. 69] является описание матрица знаний и вывод прецедента, основаный на метод логического вывода 
максимин. Результаты представленной статьи отличаются от [3, с. 62-73] тем, что выбор прецедента основывается на метод логического вывода минимакс.

Допустим, имеем матрицу знаний по прецедентам, которая была представлена в работе [3, с. 62].

Прежде всего, представим алгоритм определения функции принадлежности $\mu_{\mathrm{dj}}$ $\left(\mathrm{x}_{1}, \ldots \mathrm{x}_{\mathrm{i}}, \ldots \mathrm{x}_{\mathrm{n}}\right)$ прецедента $\mathrm{d}_{\mathrm{j}}$, интерпретируемого как нечёткое множество на универсальном множестве. $\mathrm{Ud}=\mathrm{Ux}_{1} \times \ldots \times \mathrm{Ux}_{\mathrm{i}} \times \ldots \times \mathrm{Ux}_{\mathrm{n}}$, где $\mathrm{Ux}_{\mathrm{i}}-$ универсальное множество, на котором заданы термы лингвистической переменной $\mathrm{x}_{\mathrm{i}}$, a $\mathrm{Ud}$ - декартово произведение универсальных множеств $\mathrm{Ux}_{\mathrm{i}}$.

Рассмотрим принадлежности нечёткого множества, которое образовано следующим отношением $\mu_{\mathrm{a}_{1}{ }^{\mathrm{j}}}\left(\mathrm{x}_{1}\right) \vee \ldots \vee \mu_{\mathrm{a}_{\mathrm{i}}{ }^{j}}\left(\mathrm{x}_{\mathrm{i}}\right) \vee \ldots \vee \mu_{\mathrm{a}_{\mathrm{n}}{ }^{j}}\left(\mathrm{x}_{\mathrm{n}}\right)$, где через “ $\vee "$ обозначена операция “тах", или в терминах математической логики предикат конъюнкции.

Анализируя весь блок логических высказываний, относящийся к прецеденту $\mathrm{d}_{\mathrm{j}}$ (блок соответствующих строк матрицы знаний), замечаем, что они представляют собой объединение соответствующих нечётких множеств, образовавшихся при рассмотрении строк выделенного блока. Функция принадлежности этого объединения, отождествляемая с функцией принадлежности прецедента $d_{j}$, в соответствии с [7], будет:

$$
\mu_{d_{j}}\left(x_{1}, \ldots, x_{n}\right)=\left(\mu_{\mathrm{a}_{1}^{\mathrm{j} 1}}\left(\mathrm{x}_{1}\right) \vee \ldots \vee \mu_{\mathrm{a}_{\mathrm{n}}^{\mathrm{jl}}}\left(\mathrm{x}_{\mathrm{n}}\right)\right) \wedge \ldots \wedge\left(\mu_{\mathrm{a}_{1}^{\mathrm{j} \mathrm{K}_{\mathrm{j}}}}\left(\mathrm{x}_{1}\right) \vee \ldots \vee \mu_{\mathrm{a}_{\mathrm{n}}^{\mathrm{j} \mathrm{K}_{\mathrm{j}}}}\left(\mathrm{x}_{\mathrm{n}}\right)\right)
$$

где через «^» обозначена операция “min”, или в терминах математической логики предикат дизъюнкции.

Формально представленный алгоритм определения функции принадлежности прецедента $d_{j}$ можно записать в виде:

а) фиксируем произвольную точку $\left(x_{1}^{*}, \ldots, x_{n}^{*}\right) \in U_{x_{1}} \times \ldots \times U_{x_{n}}$,

б) для каждого блока матрицы знаний, соответствующего $\mathrm{d}_{\mathrm{j}}$, определяем $\mu_{\mathrm{dj}}\left(\mathrm{x}_{1}\right.$, $\left.\ldots, \mathrm{x}_{\mathrm{n}}\right)$ в этой точке согласно схеме табл. 1.

Таблица 1.

Выбор принадлежности прецедента

\begin{tabular}{|c|c|c|c|c|c|c|}
\hline \multirow{2}{*}{$\mathrm{n} / \mathrm{n}$} & \multicolumn{2}{|l|}{ Координаты ситуационого вектора } & & & \\
\cline { 2 - 4 } & $x_{1}$ & $\ldots$ & $x_{n}$ & $\max$ & $\min$ & $d$ \\
\hline$\vdots$ & $\vdots$ & $\vdots$ & $\vdots$ & $\vdots$ & $\vdots$ & $\vdots$ \\
\hline$i_{1}$ & $\left(a_{1}^{j_{1}}\right)^{*}$ & $\cdots$ & $\left(a_{n}^{j_{1}}\right)^{*}$ & $\max _{i}\left(a_{i}^{j_{1}}\right)^{*}$ & \\
$\vdots$ & $\vdots$ & $\vdots$ & $\vdots$ & $\vdots$ & $\min _{j} \max _{i}\left(a_{i}^{j}\right)^{*}$ & $\mu_{d_{j}}$ \\
$j_{K_{j}}$ & $\left(a_{1}^{j_{K_{j}}}\right)^{*}$ & $\ldots$ & $\left(a_{n}^{j_{K_{j}}}\right)^{*}$ & $\max _{i}\left(a_{i}^{j_{K_{j}}}\right)^{*}$ & & \\
\hline$\vdots$ & $\vdots$ & $\vdots$ & $\vdots$ & $\vdots$ & $\vdots$ & $\vdots$ \\
\hline
\end{tabular}

Операция $\min a_{i}^{j s}$ производится над числами, стоящими в строках “ $i$ ”, $1 \leq i \leq n$ и в столбец "min" заносится минимальное число в соответствующей строке. Операция $\min _{j} \max \left(a_{i}^{j}\right)^{*}$ выделяет среди полученных строчных минимумов $1 \leq j_{s} \leq K_{j}$ наибольший.

Это число и является значением функции принадлежности $\mu_{d j}\left(x_{1}, \ldots, x_{n}\right)$ в этой фиксированной точке $\left(x_{1}^{*}, \ldots, x_{n}^{*}\right)$. Проведя такие вычисления для каждой точки универсального множества, получим интересующие нас функции принадлежности. 
На основе текущих измерений точка $\left(x_{1}^{*}, \ldots, x_{n}^{*}\right)$ формируется с количественными значениями его координат. Только в этой фиксированной точке $\left(x_{1}^{*}, \ldots, x_{n}^{*}\right)$ в момент поступления замера и нужно определить значение функции $\mu_{d j}\left(x_{1}, \ldots, x_{n}\right)$.

При наблюдении ситуационного вектора с количественными координатами (все координаты вектора измерены по числовым шкалам) для выбора наиболее подходящего прецедента вовсе нет необходимости полностью определять функции принадлежности $\mu_{d j}\left(x_{1}, \ldots, x_{n}\right)$ на всем множестве точек универсального множества. Достаточно рассчитать их значение только для фиксированных числовых значений координат вектора, который мы получили в результате наблюдения. Для этого придется однократно воспользоваться алгоритмом беря в качестве $\left(x_{1}^{*}, \ldots, x_{n}^{*}\right)$ координаты наблюдённого ситуационного вектора.

В результате для каждого прецедента $\mathrm{d}_{\mathrm{j}}$ мы получим число $d_{j}\left(x_{1}^{*}, \ldots, x_{n}^{*}\right)$, являющееся степенью принадлежности $d_{j}$ точке $\left(x_{1}^{*}, \ldots, x_{n}^{*}\right)$.

Исходя из такой интерпретации, наиболее предпочтительным прецедентом для разрешения наблюдаемой ПрС/С будет прецедент $\mathrm{d}_{\mathrm{j}}^{*}$, для которого

$$
d_{j}^{*}\left(x_{1}^{*}, \ldots, x_{n}^{*}\right)=\min _{1 \leq j \leq p} d_{j}\left(x_{1}^{*}, \ldots, x_{n}^{*}\right) .
$$

В отличие от [5, с. 53] в оперативно реализованном механизме вывода по прецеденте нет необходимости «принудительно» вводить метрику в алгоритм предпочтения прецедентов.

Таким образом, в идею алгоритма входит метод минимакса - правило принятия решений, используемое в теории игр, теории принятия решений, исследовании операций, статистике и философии, см, например [6, 149; 7, с. 144; 8, с. 45].

Систему работы RFID можно представить как процесс, посредством которого собираются данные в определенный момент времени. Причем не редко встречаются ситуации, когда в определенный момент времени одновременно поступают данные от $n$ меток (см.[1, с. 35]), случайным образом перекрывающих друг друга.

Согласно работ [9, с. $2 ; 10$, с. 92], поведенческая модель радиоприемного тракта ( $\mathrm{RX}$ chain) состоит из Verilog-модулей, которые реализуют процессы детектирования поднесущей, фильтрации, усиления и детектирования входного высокочастотного сигнала, представленного в языке Verilog 16-разрядным двоичным сигналом. Иными словами, что сигнал по форме является вектором х объема 16, связанных с влиянием перекрывающих сигналов. То есть на искажение влияют $n$ перекрывающихся сигналов. Допустим, что истинный сигнал представим в виде вектора $\mathbf{l}_{0}$, на который наложили искажение $\mathbf{u}$, состоящее их $n$ сигналов (векторов), принимающие значения из множества $\mathbf{l}_{1}, \mathbf{l}_{2}, \ldots, \mathbf{l}_{d}$.

Пусть поступают данные от $n$ меток, поступающие в определенный момент времени одновременно, описываются ситуационным вектором с координатами $\left\{x_{1}\right.$, $\left.x_{2}, \ldots, x_{n}\right\}$, представленными лингвистическими переменными: $x_{1}$ - энергетика сигнала от метки №1; $x_{2}$ - энергетика сигнала от метки №2; и так далее; $x_{n}$ - энергетика сигнала от метки № $n$. Пусть для этого класса наблюдались два прецедента $d_{1}, d_{2}$, каждый из которых применялся в двух (разных) случаях. Лингвистические переменные принимают следующие унифицированные значения (термы): $x_{i}=\{$ статический низкий уровень, статический высокий уровень $\}, i=1, \ldots, n$.

Пуст каждый терм лингвистических переменных представлен унифицированным универсальным множеством (десятибалльная шкала) и унифицированными кусочнолинейными (треугольными) функциями принадлежности. Допустим, например, матрица знаний для этой системы представлена в табл.2, при $n=3$. 
Таблица 2.

Пример выбора принадлежности прецедента

\begin{tabular}{|c|c|c|c|c|c|}
\hline \multirow{2}{*}{$№$} & \multicolumn{2}{|c|}{ Координаты ситуационного вектора } & $\max$ & $\min$ \\
\cline { 2 - 3 }$\Pi / \Pi$ & $\mathrm{x}_{1}$ & $\mathrm{x}_{2}$ & $\mathrm{x}_{3}$ & & \\
\hline 1.1 & 0.6 & 0.8 & 0.4 & 0.8 & 0.8 \\
1.2 & 0.6 & 0.2 & 0.8 & 0.8 & \\
1.3 & 0.6 & 0.8 & 0.6 & 0.8 & \\
1.4 & 0.9 & 0.8 & 0.8 & 0.9 & \\
\hline 2.1 & 0.4 & 0.4 & 0.5 & 0.5 & 0.5 \\
2.1 & 0.6 & 0.2 & 0.6 & 0.6 & \\
\hline
\end{tabular}

Вывод: наиболее предпочтительным прецедентом для разрешения ПрС/С ( $x_{1}^{*}=0,4, x_{2}^{*}=0,4, x_{3}^{*}=0,6$ ) является прецедент $\boldsymbol{d}_{2}$.

Таким образом, в настоящей работе сформулирован алгоритм оперативного вывода по прецеденту с применением в управлении интегральными схемами .

\section{References:}

1. Филлипенко И.В. Математическая модель систем радиочастотной идентификации с кодовым разделением каналов // Восточно-Европейский журнал передовых технологий. 2011. 5/3 (53). С. 34- 36.

2. Черняховская Л. Р., Федорова Н.И., Низамутдинова Р.И. Интеллектуальная поддержка принятия решений в оперативном управлении деловыми процессами предприятия // Вестник УГАТУ. Серия управление, вычислительная техника, информатика. Уфа: УГАТУ. 2011. Т. 15, № 2(42). С.172-176.

3. Прохоров М. Д. Федунов Б.Е. Вывод по прецеденту в базах знаний бортовых интеллектуальных систем, размещаемых на борту антропоцентрических объектов // Искусственный интеллект и принятие решений. 2010/03. С. 62-73.

4. Дшхунян В.Л. Электронная идентификация. Бесконтактные электронные идентификаторы и смарт-карты / В.Л. Дшхунян, В.Ф. Шаньгин. М.: ООО «Издательство АСТ»: Издательство «НТ Пресс», 2004. 695 с.

5. Варшавский П.Р., Еремеев А.П. Моделирование рассуждений на основе прецедентов в интеллектуальных системах поддержки принятия решений. // Искусственный интеллект и принятие решений». №2. 2009. стр. 45-57.

6. Блэкуэлла Д., Гиршик М.А. Теория игр и статистических решений / пер. с англ. И.В. Соловьева. под ред. Б. А. Севастьянова М.: изд. иностр. лит. 1958. 374 с.

7. Демьянов В.Ф., Малоземов В.Н. Введение в минимакс. М.: Наука, 1972. 368 с.

8. Godonoaga A., Balan P. A probabilistic method for solving minimax problems with general constraints // Bul. Acad. Ştiinţe Repub. Mold. Mat., 2010, номep 1, C. 33-46

9. Галев А.В., Косолапов А.С. Исследование влияния структурных помех на помехоустойчивость систем с широкополосными шумоподобными сигналами при когерентном приеме // Электронное научно-техническое издание Наука и образование. 2012, №4, апрель. С.1-15.

10. Вертегел В.В. Моделирование системы радиочастотной идентификации // Вісник СевНТУ: зб. наук. пр. Вип. 131/2012. Серія: Інформатика, електроніка, зв'язок. Севастополь, 2012. С. 95-101. 